\title{
Variação das propriedades tecnológicas de painéis MDF em uma linha de produção industrial no Brasil
}

\author{
Variation of technological properties of MDF panels in an industrial production line in Brazil \\ Variación de las propiedades tecnológicas de los paneles de MDF en una línea de producción \\ industrial en Brasil
}

Recebido: 27/08/2021 | Revisado: 02/09/2021 | Aceito: 04/09/2021 | Publicado: 06/09/2021

\author{
Elesandra da Silva Araujo \\ ORCID: https://orcid.org/0000-0002-8295-1891 \\ Universidade Federal de Lavras, Brasil \\ E-mail: elesandra.florestal@gmail.com \\ Thiago de Paula Protásio \\ ORCID: https://orcid.org/0000-0002-5560-8350 \\ Universidade Federal Rural da Amazônia, Brasil \\ E-mail: depaulaprotasio@gmail.com \\ Antonio Vinicius Correa Barbosa \\ ORCID: https://orcid.org/0000-0002-9961-2485 \\ Universidade Federal Rural da Amazônia, Brasil \\ E-mail: profvinibarbo@gmail.com \\ Rafael Farinassi Mendes \\ ORCID: https://orcid.org/0000-0002-1530-8388 \\ Universidade Federal de Lavras, Brasil \\ E-mail: rafael.mendes@ufla.br \\ José Benedito Guimarães Júnior \\ ORCID: https://orcid.org/0000-0002-9066-1069 \\ Universidade Federal de Lavras, Brasil \\ E-mail: jose.guimaraes@ufla.br \\ Lourival Marin Mendes \\ ORCID: https://orcid.org/0000-0001-8713-405X \\ Universidade Federal de Lavras, Brasil \\ E-mail:lourival@ufla.br \\ Marcela Gomes da Silva \\ ORCID: https://orcid.org/0000-0003-4928-7914 \\ Universidade Federal Rural da Amazônia, Brasil \\ E-mail: marcela.gsilva@gmail.com
}

\begin{abstract}
Resumo
No presente estudo foram caracterizadas as propriedades tecnológicas de painéis de fibras de média densidade (MDF), em diferentes horários de produção em uma linha industrial brasileira, com o objetivo de avaliar a variabilidade tecnológica das chapas e sua qualidade em relação à norma técnica comercial. Painéis MDF confeccionados com fibras de Eucalyptus spp. (70\%), Schizolobium parahyba (20\%) e madeiras residuais de serraria (10\%), aglutinados com resina ureia-formaldeído foram coletadas em intervalos de duas horas de um turno de produção da empresa. Foram determinadas às propriedades físicas (umidade, densidade aparente, absorção superficial, absorção de água, inchamento em espessura e taxa de não retorno de espessura) e resistência mecânica (flexão estática, dureza janka, tração perpendicular e arrancamento de parafuso). Os painéis de início e final do turno foram submetidos ao ciclo de envelhecimento acelerado, sendo avaliados posteriormente à densidade, tração e flexão estática. Do total das propriedades avaliadas nos painéis $\mathrm{MDF}$, um percentual de 55\% não apresentaram variações entre os horários de produção, e se enquadraram aos valores da norma. Apenas as propriedades de inchamento em espessura (IE 24h) e tração em $80 \%$ dos horários, não atenderam aos valores estipulados pela ABNT NBR 15316-1, variando de 11,2 a $15,6 \%$ e 0,42 a $0,62 \mathrm{~N} / \mathrm{mm}^{2}$, respectivamente, entre o início e o final do turno. O envelhecimento acelerado promoveu diminuição expressiva na qualidade das chapas. Os painéis MDF estudados apresentam bom desempenho tecnológico para uso em ambiente seco, e sua variação tecnológica decorreu do uso de fibras de diferentes madeiras na produção das chapas.
\end{abstract}

Palavras-chave: Painel de fibras de média densidade; Painéis comerciais; Propriedades físico-mecânicas; Envelhecimento artificial.

\footnotetext{
Abstract

In the present study the technological properties of medium density fibreboard (MDF) were characterised, at different production times in a Brazilian industrial line, with the aim of assessing the technological variability of the boards and
} 
their quality in relation to the commercial technical standard. MDF panels made with fibers from Eucalyptus spp. (70\%), Schizolobium parahyba (20\%) and sawmill waste wood (10\%), bonded with urea-formaldehyde resin were collected at two-hour intervals from one of the company's production shifts. Physical properties (moisture, bulk density, surface absorption, water absorption, thickness swelling and thickness non-return rate) and mechanical strength (static bending, janka hardness, perpendicular traction and screw pull-out) were determined. The panels from the beginning and end of the shift were submitted to the accelerated ageing cycle, and then evaluated for density, tensile and static bending. Of the total properties assessed in the MDF panels, a percentage of $55 \%$ showed no variations between the production times, and conformed to the values of the standard. Only the properties of swelling in thickness (IE 24h) and traction in 80\% of the times, did not meet the values stipulated by ABNT NBR 15316-1, ranging from 11.2 to $15.6 \%$ and 0.42 to $0.62 \mathrm{~N} / \mathrm{mm}^{2}$, respectively, between the beginning and end of the shift. Accelerated ageing caused a significant decrease in the quality of the sheets. The MDF panels studied present good technological performance for use in dry environments, and their technological variation was due to the use of fibres from different woods in the production of the boards.

Keywords: Medium density fiberboard; Commercial panels; Physical-mechanical properties; Artificial aging.

\section{Resumen}

En el presente estudio se caracterizaron las propiedades tecnológicas de los tableros de fibra de densidad media (MDF), en diferentes tiempos de producción en una línea industrial brasileña, con el objetivo de evaluar la variabilidad tecnológica de los tableros y su calidad en relación con la norma técnica comercial. Paneles de MDF fabricados con fibras de Eucalyptus spp. (70\%), Schizolobium parahyba (20\%) y residuos de madera de aserradero $(10 \%)$, aglutinados con resina de urea-formaldehído, se recogieron en intervalos de dos horas en uno de los turnos de producción de la empresa. Se determinaron las propiedades físicas (humedad, densidad aparente, absorción superficial, absorción de agua, hinchazón del espesor y tasa de no retorno del espesor) y la resistencia mecánica (flexión estática, dureza Janka, tracción perpendicular y extracción de tornillos). Los paneles del principio y del final del turno se sometieron al ciclo de envejecimiento acelerado, y luego se evaluó la densidad, la tracción y la flexión estática. Del total de propiedades evaluadas en los tableros MDF, un porcentaje del 55\% no mostró variaciones entre los tiempos de producción, y se ajustó a los valores de la norma. Sólo las propiedades de hinchamiento en el espesor (IE 24h) y de tracción en el 80\% de los tiempos, no cumplieron los valores estipulados por la ABNT NBR 15316-1, oscilando entre 11,2 y $15,6 \%$ y 0,42 y $0,62 \mathrm{~N} / \mathrm{mm}^{2}$, respectivamente, entre el inicio y el final del turno. El envejecimiento acelerado provocó una importante disminución de la calidad de las tablas. Los tableros MDF estudiados presentan un buen rendimiento tecnológico para su uso en ambiente seco, y su variación tecnológica es resultado del uso de fibras de diferentes maderas en la producción de los tableros.

Palabras clave: Tableros de fibra de densidad media; Paneles comerciales; Propiedades físico-mecánicas; Envejecimiento artificial.

\section{Introdução}

O Painel de fibras de média densidade (MDF - Medium Density Fiberboard) é atualmente a principal chapa de madeira reconstituída comercializada no Brasil. É produzida a partir de fibras de madeira, que em processo contínuo são coladas com adesivo sintético, geralmente a ureia-formaldeído, e consolidados através de prensagem a quente, sob ação controlada de calor e pressão (Indrayani et al., 2014; Iwakiri \& Trianoski, 2020). Segundo a Indústria Brasileira de Árvores (Ibá, 2020), no Brasil, encontram-se um total de 15 unidades produtoras de painéis MDF, concentradas em aproximadamente 87\% nas regiões do Sul e Sudeste. A produção dessas chapas em 2018 atingiu 3,2 milhões de metros cúbicos, um aumento de 3,2\% em relação ao ano de 2017 (Ibá, 2019).

O MDF tem sido considerado atualmente, um painel popular com ampla aplicação para usos estruturais e não estruturais, com destaque na confecção de móveis (Santos et al., 2020). Ademais, o bom desempenho dessas chapas para móveis são atribuídas à sua resistência, durabilidade, e preços mais baixos, em comparação a de madeiras maciças clássicas (Santos et al., 2019). Além disso, os painéis de fibras apresentam superfície homogênea que recebe melhor processamento e acabamentos, qualidades dificilmente encontradas em outros painéis, como nos particulados, por exemplo.

A manufatura de painéis de qualidade depende de inúmeras variáveis, que estão principalmente relacionadas à matéria-prima, tipo de adesivo e produtos aditivos incorporados aos painéis durante a fabricação (Melo et al., 2018). Desta forma, todo o processo produtivo é monitorado e, algumas das chapas do lote de produção têm suas propriedades tecnológicas 
inspecionadas no laboratório das empresas, e seus resultados determinam a classe e, por conseguinte, o valor comercial dos painéis.

Os estudos mais recentes sobre a qualidade dos painéis MDF comerciais produzidos no Brasil foram realizados por Torquato et al. (2010) e Wandscheer et al. (2016). No primeiro estudo, os autores compararam a qualidade de painéis MDF de quatro indústrias brasileiras, e observaram que as diferenças nas propriedades tecnológicas das chapas são principalmente decorrentes das variações de matéria-prima e do processo produtivo. Wandscheer et al. (2016), avaliaram o efeito do tratamento térmico em estufa na melhoria da estabilidade dimensional de painéis MDF comerciais, e concluíram que as temperaturas de 160 e $180{ }^{\circ} \mathrm{C}$, por um período de 6 e 12 min., não melhoraram a variação dimensional, assim como, não influenciaram sobre as propriedades mecânicas. Outra temática muito importante e pouco relatada na literatura é sobre a variabilidade nas propriedades tecnológicas das chapas em linha de produção industrial.

A produção de painéis de madeira em linha industrial, com menor oscilação nas propriedades físico-mecânicas, significa maior controle das variáveis envolvidas no processo produtivo e, consequentemente, a garantia de um produto de qualidade e competitivo no mercado. Neste sentido, estudos que busquem avaliar o comportamento tecnológico das chapas, em diferentes horários de fabricação, podem auxiliar na identificação de possíveis fontes de variações tecnológicas e na melhoria do processo industrial. Desta maneira, foram caracterizadas as propriedades físicas e mecânicas de painéis MDF em diferentes horários de produção em uma linha industrial brasileira, com o objetivo de avaliar a variabilidade tecnológica das chapas e sua qualidade em relação à norma vigente NBR 15316-1(ABNT, 2014).

\section{Material e Métodos}

\subsection{Obtenção e caracterização físico-mecânica dos painéis}

O estudo foi realizado em uma fábrica de painéis MDF no Brasil. Cinco chapas de MDF in natura do tipo Standard com densidade aparente entre 651 a $800 \mathrm{~kg} \mathrm{~m}^{-3}$ e dimensões finais de $2,75 \mathrm{~m}$ x 1,83 m x $15 \mathrm{~mm}$ (comprimento x largura $\mathrm{x}$ espessura) foram coletadas em um turno de produção da empresa, em intervalos de duas horas, especificamente nos horários de 7, 9, 11, 13 e 15 h. As chapas foram confeccionadas com fibras de Eucalyptus spp. (70\%), Schizolobium parahyba (20\%) e madeiras residuais de serraria (10\%), aglutinadas com resina ureia-formaldeído.

Na Tabela 1, constam as propriedades físico-mecânicas avaliadas, quantidades e dimensões dos corpos de prova, assim como, suas respectivas normas e valores de referência. Antes da realização dos ensaios, as amostras foram acondicionadas em sala de climatização à temperatura de $20 \pm 2{ }^{\circ} \mathrm{C}$ e umidade relativa de $65 \pm 5 \%$ até equilíbrio.

Tabela 1. Propriedades tecnológicas e parâmetros utilizados na caracterização dos painéis MDF.

\begin{tabular}{|c|c|c|c|c|}
\hline Propriedades físico-mecânicas & $\begin{array}{c}\mathbf{N}^{0} \text { de } \\
\text { amostras }\end{array}$ & $\begin{array}{c}\text { Dimensões } \\
(\mathbf{m m})\end{array}$ & Norma & Referência \\
\hline $\begin{array}{l}\text { Umidade } \\
\text { Densidade aparente } \\
\text { Inchamento em espessura } 2 \mathrm{~h} \text { e } 24 \mathrm{~h} \\
{ }^{1} \text { Absorção Superficial }(\mathrm{mm}) \\
\text { Arranque de parafuso face e topo }\left(\mathrm{N} / \mathrm{mm}^{2}\right) \\
\text { Tração } \\
{ }^{1} \text { Módulo de elasticidade - MOE } \\
{ }^{1} \text { Módulo de ruptura- MOR }\end{array}$ & $\begin{array}{l}5 \\
5 \\
5 \\
5 \\
3 \\
4 \\
5 \\
5\end{array}$ & $\begin{array}{c}50 \times 50 \\
50 \times 50 \\
50 \times 50 \\
500 \times 100 \\
50 \times 50 \\
50 \times 50 \\
350 \times 50 \\
350 \times 50\end{array}$ & ABNT (2014) & $\begin{array}{r}4 \text { a } 11 \% \\
0,6 \text { a } 0,8 \mathrm{~g} \mathrm{~cm}^{-3} \\
\leq 12 \% \\
\mathrm{NC} \\
\mathrm{NC} \\
\geq 0,55 \mathrm{~N} / \mathrm{mm}^{2} \\
\geq 2.200 \mathrm{~N} / \mathrm{mm}^{2} \\
\geq 20 \mathrm{~N} / \mathrm{mm}^{2}\end{array}$ \\
\hline $\begin{array}{l}\text { Absorção de água (AA\%) } \\
\text { Taxa de não retorno de espessura (TNRE\%) }\end{array}$ & 5 & $50 \times 50$ & ASTM (2002) & $\mathrm{NC}$ \\
\hline Dureza Janka & 5 & $50 \times 50$ & ABNT (2006) & $\mathrm{NC}$ \\
\hline
\end{tabular}

Em que: ${ }^{(1)}$ Ensaio executado somente nos painéis coletados em 7 e $15 \mathrm{~h} . \mathrm{NC}=$ não consta valor de referência na norma. Fontes: ABNT (2014); ASTM (2002); ABNT (2006). 


\subsection{Envelhecimento artificial}

Foi realizado o ciclo D-1 de envelhecimento acelerado da norma APA PRP 108 (APA, 1994) nos painéis MDF coletados nos horários de $7 \mathrm{e} 15 \mathrm{~h}$. As amostras foram imersas em água a $66^{\circ} \mathrm{C} \mathrm{em}$ banho-maria durante $8 \mathrm{~h}$, em estufa a $82{ }^{\circ} \mathrm{C}$ por $14,5 \mathrm{~h}$ e aclimatizadas a $20 \pm 2{ }^{\circ} \mathrm{C}$ e $65 \pm 5 \%$ de umidade relativa durante $1 \mathrm{~h} 30$, respectivamente. Após esses procedimentos, as amostras foram avaliadas em quintuplicata quanto à densidade aparente, tração e flexão estática (MOE e MOR).

\subsection{Análise estatística}

As análises estatísticas foram realizadas em um delineamento inteiramente casualizado, com cinco períodos de coleta dos painéis na linha de produção $(7,9,11,13$ e 15h) e um total de cinco, quatro e três repetições para as propriedades físicomecânicas avaliadas (Tabela 1). As propriedades de absorção superficial, dureza Janka e flexão estática, foram avaliadas somente nos horários de 7 e 15h, assim como, as propriedades avaliadas nas amostras submetidas ao ensaio de envelhecimento acelerado. Os dados foram submetidos ao teste de Shapiro-Wilk para averiguar a normalidade. As médias foram comparadas pelo teste de Scott-Knott em nível de significância de 5\% $(\alpha=0,05)$ para avaliar o efeito do horário de produção nas propriedades dos painéis MDF. As análises foram realizadas no software Sisvar, versão (5.6) (Ferreira, 2019).

\section{Resultados e Discussão}

\subsection{Propriedades físico-mecânicas}

Os valores médios de densidade aparentes e umidade dos painéis MDF obtidos a cada duas horas na linha de produção da empresa não variaram significativamente entre os horários analisados (Figura 1). Os valores dessas propriedades físicas se enquadraram nos intervalos de 4 a $11 \%$ de umidade e 0,6 a $0,8 \mathrm{~g} \mathrm{~cm}^{-3}$ de densidade aparente, estabelecidos na norma brasileira vigente (NBR 15316-1: ABNT, 2014). A ausência de variação estatisticamente significativa da densidade e umidade das chapas MDF na linha de produção industrial foram resultados muito vantajosos, pois, normalmente a alta umidade e baixa densidade afetam negativamente o desempenho mecânico das chapas. Essas propriedades físicas apresentam relação direta com as aplicações e classes de qualidade dos painéis, logo, são constantemente monitoradas nas empresas de painéis de madeira. A densidade é um dos parâmetros de melhor eficiência na predição da qualidade dos painéis (Melo, 2013), sendo utilizada na classificação e indicação do uso comercial das chapas (Silva \& Gonçalves, 2007). 
Figura 1. Valores médios de densidade aparente e teor de umidade dos painéis MDF.

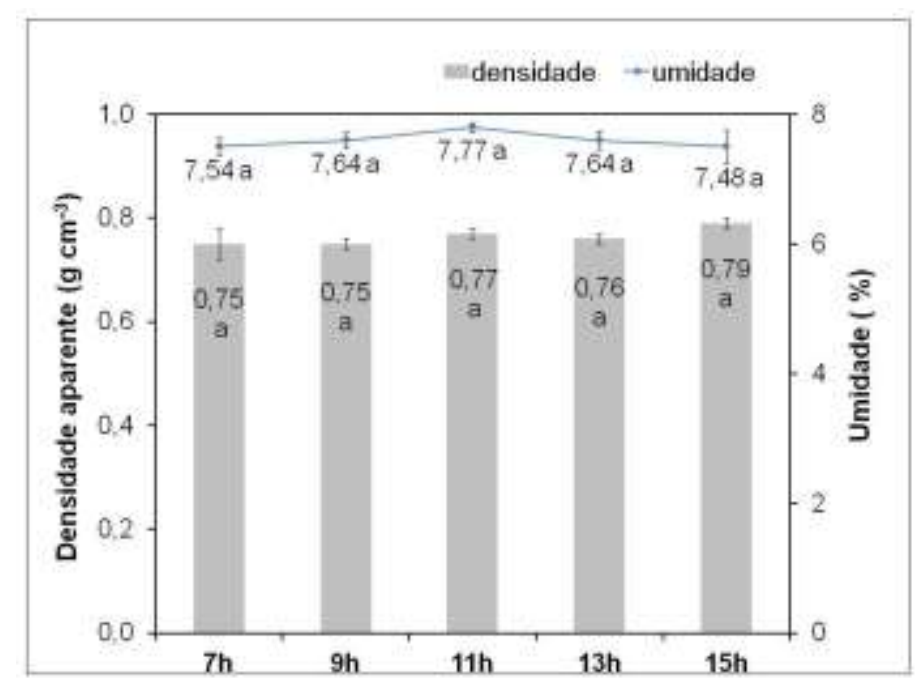

Em que: Médias seguidas pela mesma letra para cada propriedade não diferem estatisticamente pelo teste de Scott-Knott a 5\% de significância. Fonte: Autores.

O inchamento em espessura (IE) após $2 \mathrm{~h}$ em água variaram de 5,0 a 8,8\% entre os horários avaliados, enquanto as amostras imersas por $24 \mathrm{~h}$ variaram de 11,2 a $15,6 \%$, sendo que apenas os painéis de $11 \mathrm{~h}$ atenderam ao valor prescrito na norma (Tabela 2). Os valores de absorção de água (AA) após 24h variaram de 36,9 a 63,8\%, em que, os painéis de 11h apresentaram os menores valores de AA e taxa de não retorno de espessura (TNRE) (Tabela 2), indicando o menor percentual de liberação de tensões irreversíveis nestes painéis. Os maiores resultados de IE e AA 24h foram registrados para os painéis de 9 e 13h (Tabela 2), onde foi observado um acréscimo percentual médio de água de aproximadamente $79 \%$ para o IE e 55\% para a AA ao se atingir 24h de imersão. As variações no IE e AA dos painéis MDF comerciais podem ter decorrido do uso de fibras de diferentes espécies madeireiras, o que promoveu diferentes sítios higroscópios, dificultando o controle e a padronização das chapas durante o processo produtivo. Além disso, o tipo de resina pode ter favorecido a instabilidade dimensional, já que a uréia-formaldeído não é um adesivo resistente à água.

Tabela 2. Valores médios de inchamento em espessura (IE) e absorção de água (AA) após 2 e $24 h$ de imersão em água, e taxa de não retorno de espessura (TNRE) dos painéis MDF.

\begin{tabular}{lccccc}
\hline Horário (h) & IE 2h (\%) & IE 24h(\%) & AA 2h (\%) & AA 24h (\%) & TNRE (\%) \\
\hline $\mathbf{7}$ & $5,62 \mathrm{a}^{(20,7)}$ & $13,29 \mathrm{~b}^{(5,1)}$ & $25,96 \mathrm{a}^{(55,5)}$ & $50,20 \mathrm{a}^{(17,9)}$ & $4,94 \mathrm{a}^{(4,4)}$ \\
$\mathbf{9}$ & $8,78 \mathrm{~b}^{(9,9)}$ & $15,62 \mathrm{~b}^{(10,3)}$ & $40,08 \mathrm{~b}^{(29,9)}$ & $63,66 \mathrm{c}^{(9,6)}$ & $5,74 \mathrm{~b}^{(11,6)}$ \\
$\mathbf{1 1}$ & $5,14 \mathrm{a}^{(13,0)}$ & $11,20 \mathrm{a}^{(4,3)}$ & $15,50 \mathrm{a}^{(45,7)}$ & $36,88 \mathrm{~b}^{(9,7)}$ & $3,82 \mathrm{c}^{(8,4)}$ \\
$\mathbf{1 3}$ & $8,48 \mathrm{~b}^{(15,8)}$ & $15,28 \mathrm{~b}^{(17,4)}$ & $42,06 \mathrm{~b}^{(33,9)}$ & $63,78 \mathrm{c}^{(4,5)}$ & $4,58 \mathrm{a}^{(11,8)}$ \\
$\mathbf{1 5}$ & $5,04 \mathrm{a}^{(19,7)}$ & $13,94 \mathrm{~b}^{(10,4)}$ & $9,88 \mathrm{a}^{(14,3)}$ & $41,14 \mathrm{~b}^{(12,7)}$ & $4,47 \mathrm{a}^{(5,6)}$ \\
\hline Média & 6,6 & 13,9 & 26,7 & 51,1 & 4,7 \\
$\mathbf{C V ~ ( \% )}$ & 15,6 & 11,5 & 41,2 & 11,3 & 9,3 \\
\hline
\end{tabular}

Em que: Médias seguidas pela mesma letra na coluna não diferem estatisticamente pelo teste de Scott-Knott a $5 \%$ de significância. Os valores entre parênteses indicam o coeficiente de variação (CV\%). Fonte: Autores.

Vale ressaltar que o ensaio de inchamento, expressa a variação percentual do aumento em espessura da amostra após imersão em água, e um valor máximo de 12\% é prescrito para os painéis MDF na NBR 15316-1 (ABNT, 2014), enquanto, o teste de absorção nos informa o percentual de aumento de massa em água. Essas propriedades são consideradas importantes parâmetros de qualidade física, pois, possibilitam o direcionamento dos painéis para ambientes com condições severas ou mais amenas de umidade (Freire et al., 2011). Além disso, as variações referentes à perda ou ganho de umidade têm grande 
importância pela relação direta que estabelecem com as demais propriedades tecnológicas das chapas (Mendes et al., 2015), podendo gerar prejuízos, principalmente de perda de resistência e diminuição da vida útil dos painéis (Melo, 2013), com isso, precisam ser mais bem monitoradas.

Em comparação com a literatura, Belini e Tomazello Filho (2010) confeccionaram chapas de MDF em linha de produção industrial com densidade nominal de $700 \mathrm{~kg} \mathrm{~m}^{-3}$ utilizando fibras de Eucalyptus grandis e adesivo ureia-formaldeído (11\%). Os autores relataram valores de 5,8\% de inchamento em espessura $24 \mathrm{~h}$ e $33,7 \%$ de absorção de água $24 \mathrm{~h}$, evidenciando que a maior taxa de IE e AA dos painéis aqui estudados decorreram principalmente da variabilidade de fibras. Apesar disso, o fato da empresa utilizar 10\% de resíduos madeireiros provenientes de serrarias na confecção dos painéis, demonstra sua atuação em prol da sustentabilidade por meio do reaproveitamento e incorporação de resíduos florestais no processo produtivo. Desta forma, acreditamos que uma das maneiras de reduzir a variação higroscópica dos painéis seria utilizá-los com revestimento do tipo laminado baixa pressão (BP) ou finish foil (FF), os quais de certa forma promovem uma barreira física ao acesso da água.

Em relação à absorção superficial, os resultados obtidos para os painéis MDF de 7 e $15 \mathrm{~h}$ foram de 219,4 $\pm 3,7 \mathrm{~mm}$ e $234,9 \pm 1,3 \mathrm{~mm}$, respectivamente, os quais não apresentaram diferença significativa, indicando que as chapas apresentam homogeneidade nas superfícies para um bom acabamento. Além disso, esses resultados nos mostram que houve interação e compatibilidade das fibras das diferentes espécies madeireiras, que permitiu o entrelaçamento para a formação de chapas com superfícies homogêneas. Vale ressaltar, que o ensaio de absorção superficial consiste na medição do comprimento da mancha causada pelo escoamento de um solvente aplicado na superfície inclinada da amostra de painel (NBR 15316-1 ABNT, 2014), em que as maiores extensões deixadas na superfície indicam a menor absorção do produto pelo painel e vice-versa, informação importante para se avaliar o desempenho das chapas quanto à aplicação de produtos (tintas e vernizes) na fase de acabamentos, assim como, na predição dos custos com o uso desses produtos.

Os ensaios mecânicos são essenciais na determinação do comportamento dos painéis quando sujeito a ação de forças, essas informações contribuem para seu correto direcionamento em aplicações que exijam menor resistência em comparação às cargas suportadas no ensaio. Os valores médios de MOR e MOE não diferiram estatisticamente e foram superiores aos requisitos mínimos de 20 e $2.200 \mathrm{~N} / \mathrm{mm}^{2}$ previstos na norma NBR 15316-1(ABNT, 2014), respectivamente (Figura 2).

Figura 2. Valores médios de Módulo de elasticidade (MOE) e Módulo de ruptura (MOR) dos painéis MDF.

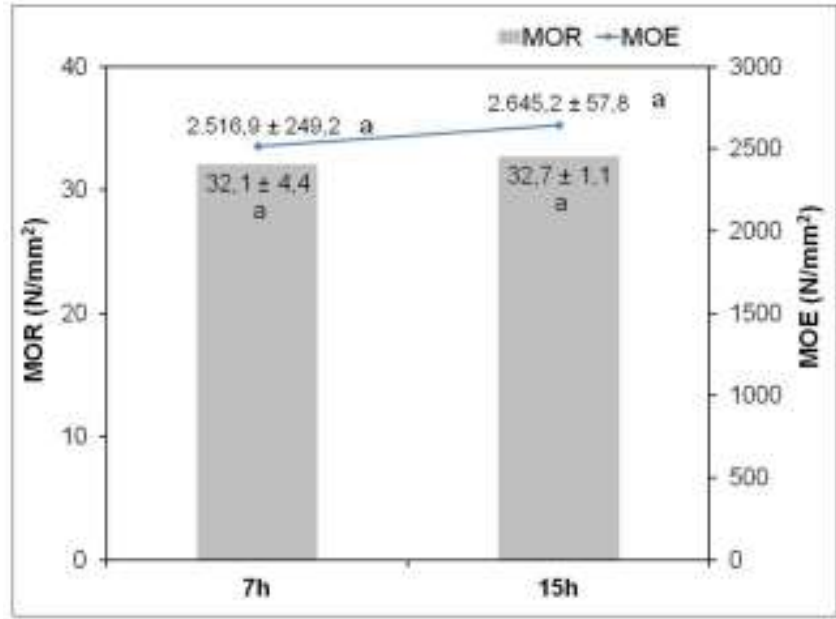

Em que: Médias seguidas pela mesma letra para cada propriedade não diferem estatisticamente pelo teste de Scott-Knott a $5 \%$ de significância. Fonte: Autores. 
Nos painéis produzidos nos horários de 7 e 15h, os valores médios de dureza Janka foram de 555,7 £52,9 Kgf e 555,0 \pm 42,9 Kgf, respectivamente. Não foi detectado efeito estatístico do horário de produção na linha industrial para esta propriedade. A dureza Janka informa à resistência que o painel oferece à penetração de outro corpo (Kravchenko \& Ferreira, 2015), característica que pode auxiliar na intensidade do processamento e trabalhabilidade das chapas na confecção de diferentes produtos.

Os valores de resistência à tração perpendicular na superfície dos painéis variaram de 0,42 a $0,62 \mathrm{~N} / \mathrm{mm}^{2}$ (Tabela 3), em que, apenas o painel de $7 \mathrm{~h}$ se enquadrou ao recomendado na norma ( $\geq 0,55 \mathrm{~N} / \mathrm{mm}^{2}$; NBR 15316-1:ABNT, 2014). Contudo, todas as chapas produzidas apresentaram resistência à tração superior ao observado por Torquato et al. (2010) para painéis MDF do tipo standard $\left(0,30 \mathrm{~N} / \mathrm{mm}^{2}\right)$, produzidos com fibras de Eucaliptus em uma fábrica no Brasil. A resistência ao arrancamento de parafuso comportou-se de forma similar à tração, com maiores valores para os painéis de 7h (FACE $=137,00$ kgf e TOPO= 94,98 kgf) e menores valores médios para os painéis coletados $11 \mathrm{~h}$ (FACE= 85,39 kgf e TOPO= 47,72 kgf). Essa resistência mecânica inferior dos painéis de $11 \mathrm{~h}$ pode ter sido ocasionada pela maior umidade, por mais que não tenha ocorrido efeito significativo entre os horários na umidade, observamos que os melhores resultados de tração e arranque de parafuso foram dos painéis de 7 e 15h (Tabela 3), os quais tiveram o menor percentual de água (Figura 1).

Tabela 3. Valores médios de tração e arranque de parafuso (AP) dos painéis MDF.

\begin{tabular}{lccc}
\hline Horário (h) & Tração $\left(\mathbf{N} / \mathbf{m m}^{\mathbf{2}}\right)$ & AP face (kgf) & AP topo (kgf) \\
\hline $\mathbf{7}$ & $0,62 \mathrm{a}^{(11,7)}$ & $137,00 \mathrm{a}^{(1,4)}$ & $94,98 \mathrm{a}^{(9,5)}$ \\
$\mathbf{9}$ & $0,46 \mathrm{~b}^{(19,2)}$ & $113,11 \mathrm{~b}^{(6,8)}$ & $62,68 \mathrm{~b}^{(31,1)}$ \\
$\mathbf{1 1}$ & $0,42 \mathrm{~b}^{(5,1)}$ & $85,39 \mathrm{c}^{(24,2)}$ & $47,72 \mathrm{~b}^{(40,9)}$ \\
$\mathbf{1 3}$ & $0,46 \mathrm{~b}^{(15,5)}$ & $107,96 \mathrm{~b}^{(11,2)}$ & $48,79 \mathrm{~b}^{(11,2)}$ \\
$\mathbf{1 5}$ & $0,52 \mathrm{~b}^{(12,5)}$ & $128,04 \mathrm{a}^{(5,6)}$ & $94,76 \mathrm{a}^{(1,6)}$ \\
\hline Média & 0,5 & 114,3 & 69,8 \\
$\mathbf{C V}(\boldsymbol{\%})$ & 14,3 & 10,5 & 22,9 \\
\hline
\end{tabular}

Em que: Médias seguidas pela mesma letra na coluna não diferem entre si a 5\% de significância pelo teste de Scott-Knott. Os valores entre parênteses indicam o coeficiente de variação (CV \%). Fonte: Autores.

De modo geral, do total das propriedades físico-mecânicas avaliadas nos painéis MDF em linha de produção industrial 55\% não apresentaram variação entre os horários avaliados, assim como, atenderam aos valores previstos na norma (Tabela 4). Dentre os ensaios que constam valores de referência na norma, somente o inchamento em espessura e tração variou entre os horários de produção avaliados, não atendendo totalmente à norma. Desta forma, tornou-se evidente que a variação das propriedades entre os horários é um fator limitante na padronização das chapas quanto à qualidade prevista em norma.

Tabela 4. Relação das propriedades dos painéis quanto à variação nos horários e cumprimento da norma.

\begin{tabular}{l|c|c}
\hline \multicolumn{1}{c|}{ Propriedades físico-mecânicas } & Variou nos horários & Atendeu à norma \\
\hline Umidade & Não & Sim \\
Densidade aparente & Não & Sim \\
Inchamento em espessura (IE) & Sim & Parcial \\
Absorção de água (AA) & Sim & NC \\
Taxa de não retorno de espessura (TNRE) & Sim & NC \\
Absorção Superficial & Não & NC \\
Módulo de elasticidade (MOE) & Não & Sim \\
Módulo de ruptura (MOR) & Não & Sim \\
Dureza Janka & Não & NC \\
Tração & Sim & Parcial \\
Arranque de parafuso face e topo & Sim & NC \\
\hline
\end{tabular}

Em que: $\mathrm{NC}=$ não consta valores de referência na norma. Fonte: Autores. 


\subsection{Resistência ao envelhecimento artificial}

Os valores médios de densidade aparente dos painéis MDF, antes e após o ensaio de envelhecimento não variaram entre os horários avaliados, contudo, o envelhecimento artificial promoveu um decréscimo médio de 37,7\% no desempenho dessa propriedade física (Figura 3A). A tração variou significativamente entre os horários nos painéis sem envelhecimento, com valor maior e dentro da norma para os painéis de $7 \mathrm{~h}$, sendo observado no geral, uma queda de $98,8 \%$ na qualidade das chapas para esse parâmetro (Figura 3B).

Figura 3. Valores médios de densidade aparente (A) e tração (B) dos painéis MDF com e sem o tratamento de envelhecimento acelerado.

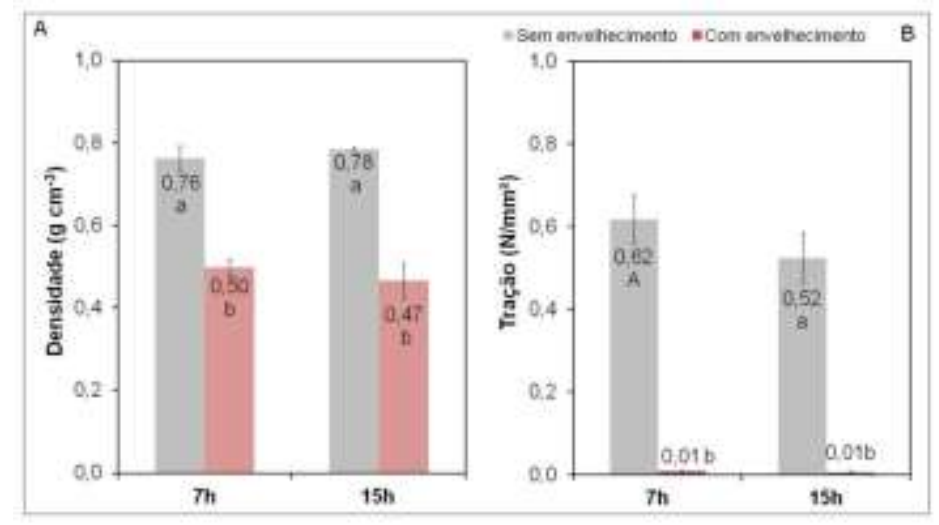

Em que: Barras da mesma cor seguidas da mesma letra não apresentam diferença entre si pelo teste Scott-Knott a 5\% de significância. Fonte: Autores.

As propriedades de módulo de elasticidade e módulo de ruptura variaram somente nos painéis envelhecidos de forma acelerada, com maior resistência dos painéis de 7h (Figura 4AB). Para ambas as propriedades foram observadas uma diminuição média de aproximadamente $98 \%$ na resistência mecânica das chapas.

Figura 4. Valores médios de Módulo de elasticidade (A) e Módulo de ruptura (B) dos painéis MDF com e sem o tratamento de envelhecimento acelerado.

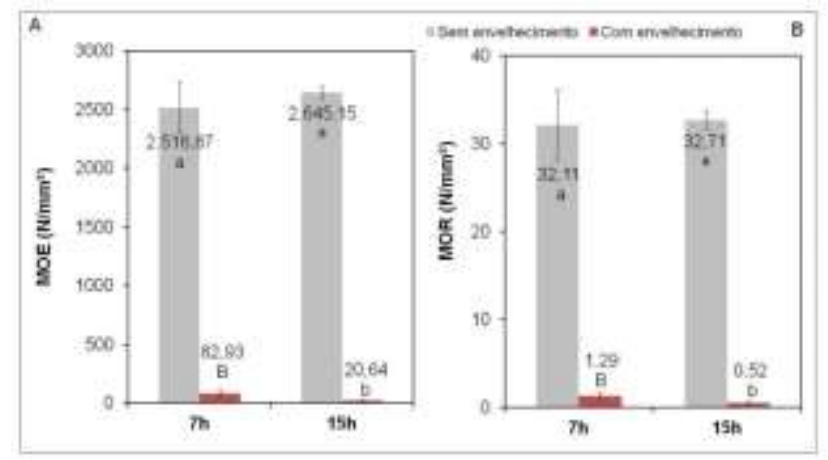

Em que: Barras da mesma cor seguidas da mesma letra não apresentam diferença entre si pelo teste Scott-Knott a 5\% de significância. Fonte: Autores.

Ressalta-se que o tratamento de envelhecimento artificial acelerado em painéis derivados de madeira avalia a influência de condições controladas de fatores de degradação, como o calor e umidade no desempenho tecnológico, permitindo a estimação da durabilidade e comportamento dos painéis ao longo do tempo por meio das mudanças em suas propriedades 
físico-mecânicas (Garzón et al., 2012; Varanda et al., 2019). O tratamento de envelhecimento artificial promoveu diminuição expressiva no desempenho dos painéis MDF de ambos os horários avaliados, em que, após o envelhecimento todas as propriedades não atenderam aos valores previstos na norma (Tabela 1). Além disso, constatamos que as perdas tecnológicas mais severas ocorreram nas propriedades mecânicas, isso decorre da influência negativa que o ganho demasiado de água exerce na resistência mecânica, principalmente por promover o afastamento das fibras. Esse fácil acesso da água pode ser explicado pela degradação do adesivo pela temperatura, haja vista que em condições normais o adesivo polimerizado pode atuar como uma barreira à água ao envolver as fibras.

\section{Conclusão}

Os painéis de fibras de média densidade (MDF) apresentam bom desempenho tecnológico para uso em ambiente seco. Apenas as propriedades de inchamento em espessura e tração em $80 \%$ dos horários avaliados, não atenderam aos valores estipulados pelo padrão normativo comercial da ABNT NBR 15316-1 (2014).

As propriedades que não variaram entre os horários de produção industrial se enquadraram aos valores da norma. A variação tecnológica se deve ao uso de fibras de diferentes espécies madeireiras na produção das chapas. Apesar disso, a utilização de $10 \%$ de mix residuais madeireiros promove sustentabilidade ao processo produtivo, sem acarretar grandes prejuízos ao desempenho tecnológico dos painéis, uma vez que a maioria das propriedades físico-mecânicas atendeu a norma comercial. Além do mais, as chapas estudadas foram do tipo in natura, assim, acreditamos que a aplicação de capa de revestimento pode reduzir essas variações tecnológicas. Desta forma, sugerimos que este estudo seja replicado em painéis revestidos com laminado baixa pressão (BP) e finish foil (FF) na mesma linha fabril.

\section{Referências}

American society testing and materials. (2002).ASTM D-1037: Standard methods of evaluating of wood-base fiber and particles materials.

Apa - the engineered wood association. (1994). PRP 108: Performance Standards and Policies for Structural-Use Panels. Washington.

Associação brasileira de normas técnicas. (2006). NBR 14810-3: Métodos de ensaios.

Associação brasileira de normas técnicas. (2014). NBR 15316-1: Painéis de fibras de média densidade. Parte 1: Terminologia.

Associação brasileira de normas técnicas. (2014). NBR 15316-2: Painéis de fibras de média densidade. Parte 2: Requisitos e métodos de ensaios.

Belini, U. L., \& Tomazello Filho, M. (2010). Avaliação tecnológica de painéis MDF de madeira de Eucalyptus grandis confeccionados em laboratório e em linha de produção industrial. Ciência Florestal, 20(3), 493-500. https://doi.org/10.5902/198050982063

Ferreira, D. F. (2019). A computer analysis system to fixed effects split plot type designs. Rev. Bras. Biometria, 37, 529-535. https://doi.org/10.28951/rbb. v37i4.450.

Freire, C. de S., Silva, D. W., Scatolino, M. V., César, A. A. da S., Bufalino, L., \& Mendes, L. M. (2011). Propriedades físicas de painéis aglomerados comerciais confeccionados com bagaço de cana e madeira. Floresta e Ambiente, 18(2), 178-185. https://doi.org/10.4322/floram.2011.036

Garzón, N., Sartori, D., Zuanetti, I., Barbirato, G., Ramos, R., Fiorelli, J., Santos, S. F., \& Savastano, H. (2012). Durability Evaluation of Agro-Industrial Waste-Based Particle Boards Using Accelerated Aging Cycling Tests. Key Engineering Materials, 517, 628-634. https://doi.org/10.4028/www.scientific.net/KEM.517.628

Indústria brasileira de árvores - IBÁ. (2019). Relatório Anual 2019.

Indústria brasileira de árvores - IBÁ. (2020). Relatório Anual 2020.

Indrayani, Y., Setyawati, D., Yoshimura, T., \& Umemura, K. (2014). Termite Resistance of Medium Density Fibreboard Produced from Renewable Biomass of Agricultural Fibre. Procedia Environmental Sciences, 20, 767-771. https://doi.org/10.1016/j.proenv.2014.03.092

Iwakiri, s.; Trianoski, R. (2020). Painéis de madeira reconstituída. (2a ed.), Fupef, 259 p.

Kravchenko, G. A., \& Ferreira, E. de M. (2015). Eficiência de diferentes adesivos para a confecção de painéis de bambu laminado. $19(1), 8$.

Melo, R. R. de, Muhl, M., Stangerlin, D. M., Alfenas, R. F., \& Rodolfo Junior, F. (2018). Propriedades de painéis aglomerados submetidos ao tratamento térmico. Ciência Florestal, 28(2), 776. https://doi.org/10.5902/1980509832109 
Research, Society and Development, v. 10, n. 11, e478101119951, 2021

(CC BY 4.0) | ISSN 2525-3409 | DOI: http://dx.doi.org/10.33448/rsd-v10i11.19951

Melo, R. R. (2013). Estabilidade Dimensional de Compostos de Madeira. Revista Ciência da Madeira - RCM, 4(2), 152-175. https://doi.org/10.12953/21776830.v04n02a03

Mendes, R. F., Mendes, L. M., Protásio, T. D. P., Oliveira, S. L., Carvalho, A. G., \& Farrapo, C. L. (2015). Umidade de equilíbrio de painéis OSB em função da umidade relativa e da temperatura ambiente. Ciência Florestal, 25(4), 1001-1014. https://doi.org/10.5902/1980509820661

Santos, P., Pitarch, J. L., \& de Prada, C. (2019). Energy-efficient Operation of a Medium Density Fibreboard Dryer Through Nonlinear MPC. IFACPapersOnLine, 52(1), 400-405. https://doi.org/10.1016/j.ifacol.2019.06.095

Santos, P., Pitarch, J. L., Vicente, A., de Prada, C., \& García, Á. (2020). Improving operation in an industrial MDF flash dryer through physics-based NMPC. Control Engineering Practice, 94, 104213. https://doi.org/10.1016/j.conengprac.2019.104213

Silva, S. A. M; \& Gonçalves, R. (2007). Avaliação da distribuição da densidade em MDF a partir da técnica da onda de ultra-som. Scientia Forestalis, 74, 1926.

Torquato, L. P., Iwakiri, S., Bonduelle, G. M., Albuquerque, C. E. C. de, \& Matos, J. L. M. de. (2010). Avaliação das propriedades físicas e mecânicas de painéis de fibras de média densidade (MDF) produzidos pelas indústrias brasileiras. Floresta, 40(2). https://doi.org/10.5380/rf.v40i2.17823

Varanda, L. D., Alesi, L. S., Yamaji, F. M., Panzera, T. H., Christoforo, A. L., \& Lahr, F. A. R. (2019). Mechanical properties of accelerated aging particleboards. Scientia Forestalis, 47 (123). https://doi.org/10.18671/scifor.v47n123.18

Wandscheer, R. B., Bressan, J., Melo, R. R., Lima, D. C., Pedrosa, T. D., \& Ferreira, M. D. (2016). Thermal treatment effect on physical and mechanical properties of MDF panels. Nativa, 4(2), 71-76. https://doi.org/10.14583/2318-7670.v04n02a03 